\title{
Case Report \\ Left accessory pathway with decremental conduction and bilateral bundle branch block and slow pathway: an unusual combination
}

\section{Introduction}

Atrioventricular reentrant tachycardia is the second most common type of reentrant supraventricular tachycardia in patients with no structural heart disease. In this case, the reentry mechanism involves the atrioventricular (AV) node and an extra nodal accessory pathway that connects the atrium with the ventricles. These accessory pathways are found along the tricuspid and mitral annulus but are very rarely located in the anterior and anteroseptal regions of the mitral annulus. Reentry occurs in most cases in an orthodromic way (the stimulus descends through the AV node and ascends through the accessory pathway) and therefore the QRS complex is narrow and shows a normal morphology. Less frequently, reentry occurs in an antidromic form.

We present a patient with no structural heart disease who attended the medical consultation with a preexcited tachycardia simulating a ventricular tachycardia originated from the left ventricular outflow tract or mitroaortic continuity.

\section{Clinical case}

We present the case of a 16-year-old patient with recurrent short episodes of paroxysmal palpitations. This last episode was more prolonged and motivated the medical consultation. On admission, the patient was hemodynamically stable and a wide QRS complex tachycardia was identified in the 12-lead EKG, which was initially interpreted as ventricular tachycardia and therefore reversed by electrical cardioversion. The 12-lead EKG in sinus rhythm and the echocardiogram showed no abnormal findings. He was referred to our institution to perform an electrophysiological study with radiofrequency ablation.

Certainly, the patient presented a regular, wide QRS complex tachycardia, with right bundle branch block morphology in precordial leads, inferior axis and positive precordial concordance (Figure 1). During the electrophysiological study, a decapolar catheter was placed in the coronary sinus, a quadripolar catheter in His, and another in a right ventricle tip. The baseline conduction times were within normal limits. During oversensing extra stimulation and with programmed atrial stimulation with decreasing cycles, progressive widening of the QRS complex was observed with left bundle branch block morphology but with a negative QRS complex in aVL and prolongation of the HV interval up to $84 \mathrm{msec}$. With even shorter cycles, the QRS complex morphology changed to right bundle branch block with greater prolongation of the HV interval up to $94 \mathrm{msec}$ (Figure 2). With programmed atrial stimulation in the proximal coronary sinus, we observed a dual AV nodal pathway physiology with a single narrow echo beats. With shorter cycles and longer AV prolongation, clinical tachycardia was induced (Figure 3). This tachycardia presented an atrio-ventricular ratio of 1 to 1 with concentric retroconduction and variable VA intervals (mostly short).

It could be terminated both by atrial and ventricular pacing and induced from the atrium and the ventricle. During the tachycardia,

\author{
Volume II Issue 2 - 2018
}

\begin{abstract}
Albina G, Giniger A, Mondragón I, Rivera S, Galizia Brito V, Caro M, Vecchio N, Jarma J, Tomas L, Scazzuso F

Buenos Aires Cardiovascular Institute, Colombia
\end{abstract}

Correspondence: Albina G, Buenos Aires Cardiovascular Institute, Colombia, Email gastonalbina@gmail.com

Received: February 09, 2018| Published: March 13, 2018

a single atrial extrastimulus activated the anterograde ventricular activation with the same morphology and the same ligature, demonstrating the participation of the accessory pathway in the reentrant circuit. With a diagnosis of antidromic AV reentrant tachycardia, the coronary sinus catheter was progressed until hour 12 , where a greater precocity to the surface QRS complex was achieved. Endocardial mapping by retrograde arterial puncture was performed using a $4 \mathrm{~mm}$ EPT catheter. By radiofrequency application with 45 watts and 58 degrees celsius of temperature, the definitive interruption of the tachycardia was achieved at hour 12, below the left valsalva and non-coronary sinuses (Figure 4). It was not possible to re-induce tachycardia and the response of the intraventricular conduction against programmed atrial stimulation until the refractory period of the AV node was normal, with disappearance of bilateral branch block.

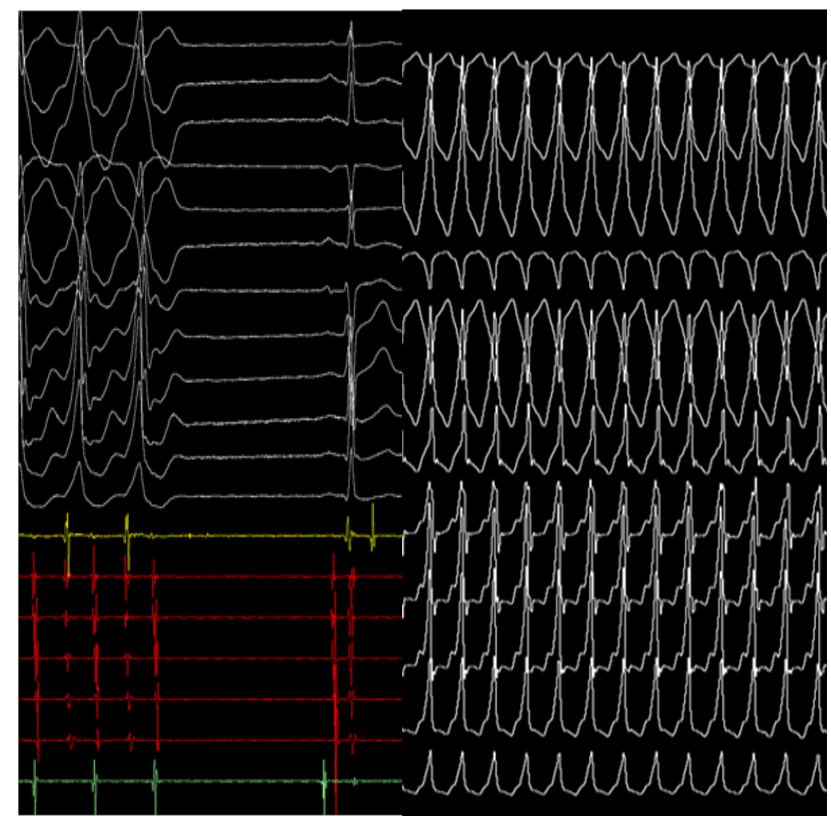

A

B

Figure I (A) Basal I2-lead electrocardiogram. (B) I2-lead electrocardiogram with wide QRS complex tachyarrhythmia. 


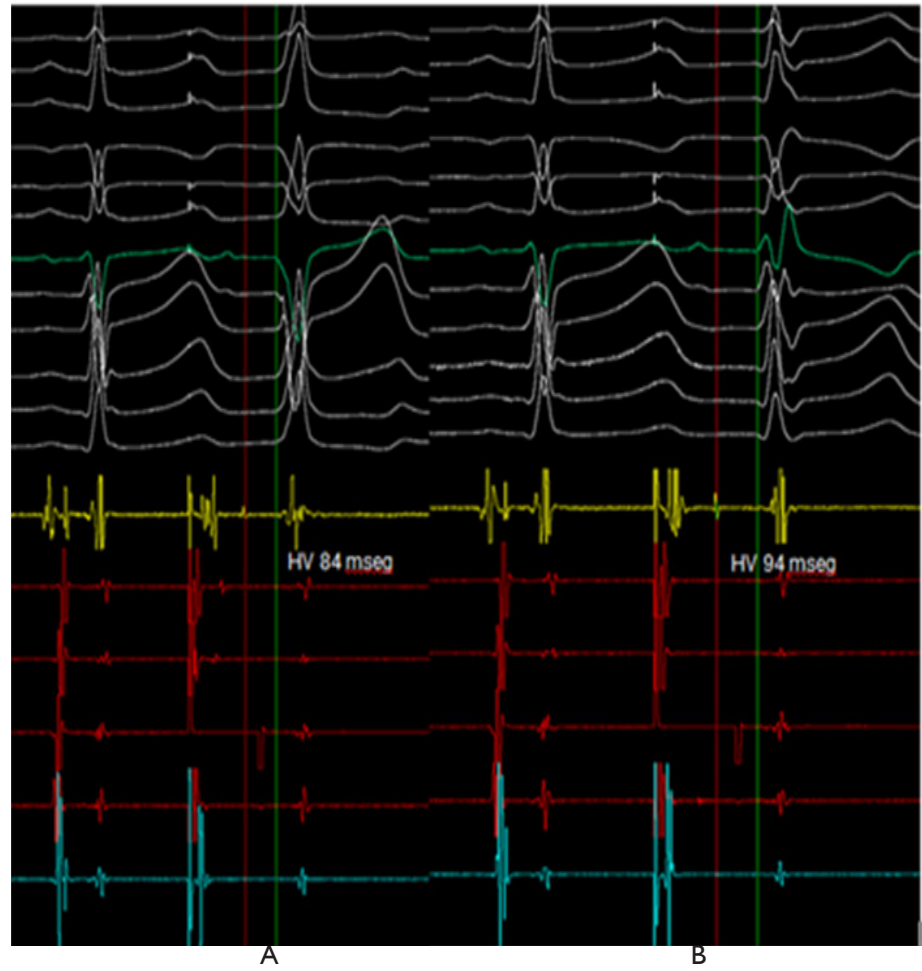

Figure 2 (A) Oversensing extra stimulation with programmed atrial stimulation, with left bundle branch block aberrant complex and prolongation of the HV interval. (B) Right bundle branch block aberrant complex and greater prolongation of the HV interval seen in shorter cycles.

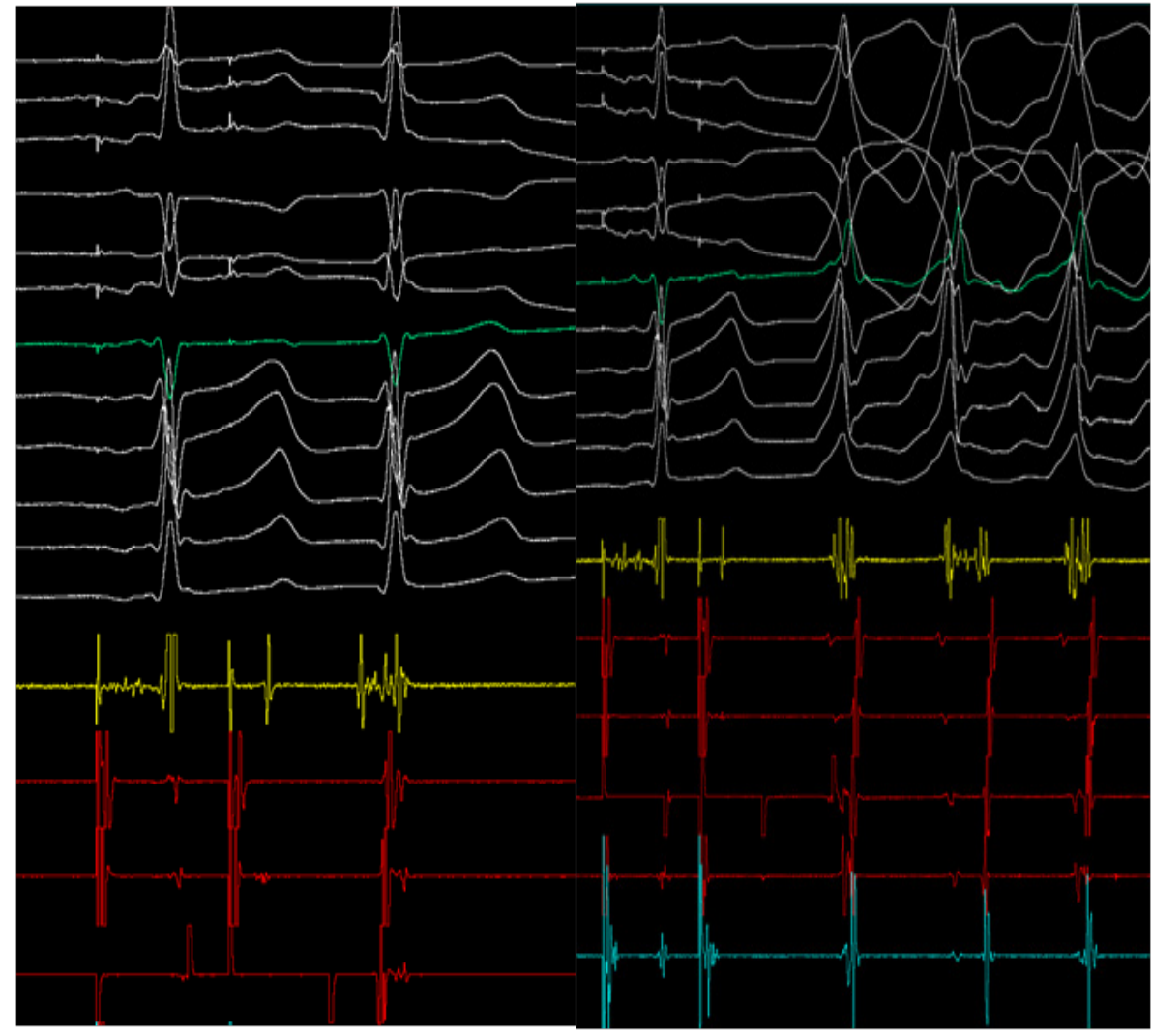

Figure 3 (A) Programmed atrial stimulation:AV jump and AV reentry with a narrow QRS complex. (B) In shorter cycles, narrow QRS complex tachycardia. 


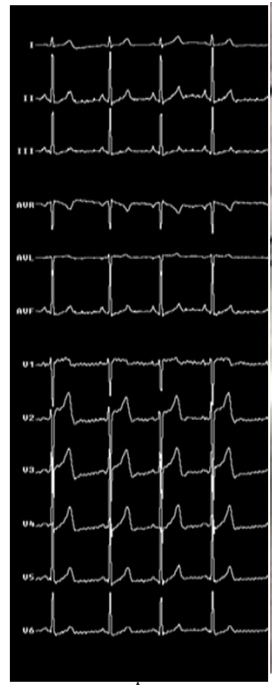

A

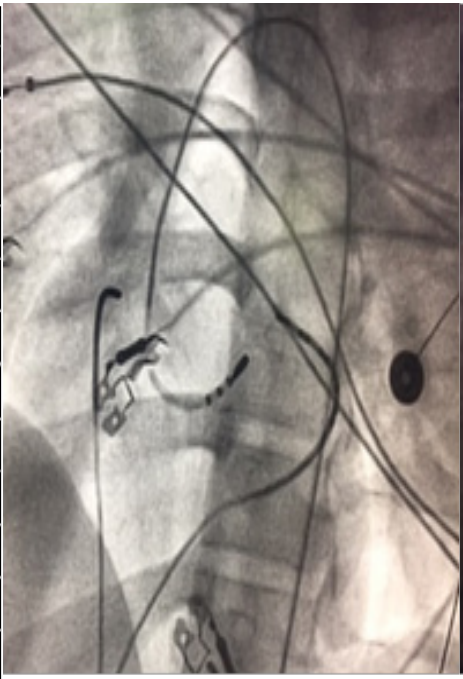

B

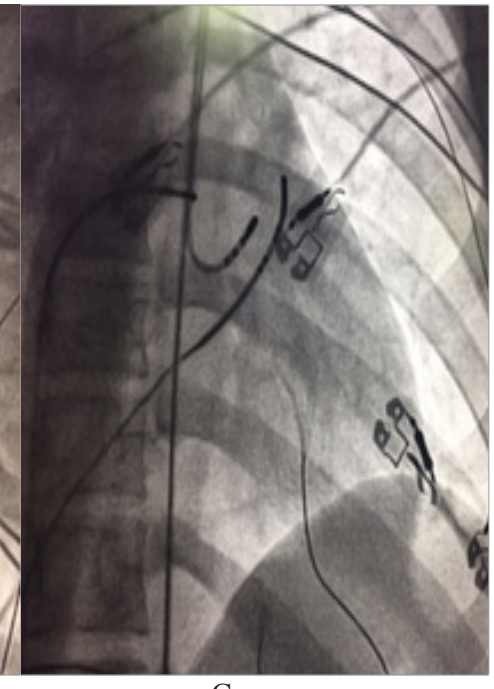

C

Figure 4 (A) Termination of tachycardia during RF application. (B) Fluoroscopy image in left anterior oblique view of the ablation site and (C) right anterior oblique view with the ablation catheter in the ablation site.

\section{Discussion}

In this case, we describe a patient with an unusual left accessory pathway with decremental conduction associated with a slow pathway that was successfully ablated in the mitroaortic continuity, below the left aortic cusp. Although there are sporadic cases of accessory pathways with this atypical location, the association with a slow pathway and modulation of the conduction pathways are extremely rare and we have found no evidence of any published case in the literature.

Accessory pathways have been classified according to different algorithms. In all of them and as in the original description of Gallagher, there is no description of accessory pathways localized in the mitral annulus between hour 8 and $1 .{ }^{1}$ The anterior and left anteroseptal pathways are extremely rare due to the presence of extensive fibrosis between the mitral valve and the aortic non-coronary and left coronary cusps. Several reports have described ablation of accessory pathways in this localization, both by transseptal and retrograde aortic approach. In most cases, patients had clear preexcitation in the 12 lead EKG and, more rarely, had hidden accessory pathways. Bradley Wilsmore and collaborators published in 2012, for the first time, a similar case of a 42-year-old patient with no evident preexcitation that presented with wide QRS complex tachycardia simulating a ventricular tachycardia originated in the outflow tract. This case was a left anterior accessory pathway with slow and decremental antegrade conduction that was revealed only with a marked delay (or blockage) of conduction in the AV node and that was ablated from the left valsalva sinus. The author of this report asociated the presence of this pathway to the development of the conduction system based on the decremental properties and on histological studies that evidenced the presence of ventricular musculature beyond the semilunar valves in a significant number of patients. ${ }^{2}$ These findings are similar to those evidenced in our patient. Indeed, our patient presented with a wide QRS complex tachycardia in which no hisian activity was recorded preceding each QRS complex, suggesting a ventricular tachycardia or a pre-excited tachycardia. The diagnosis of ventricular tachycardia was excluded by demonstrating that a single atrial extrastimulus advanced the anterograde ventricular activation with the same QRS complex morphology and because rapid atrial pacing terminated tachycardia without advancing any QRS complex (Figure 5). The tachycardia was also inducible with ventricular extrastimuli with long coupling intervals, which along with the concentric retroconduction evidenced the absence of retrograde conduction by an accessory pathway. The inducibility from the ventricle practically ruled out the diagnosis of atrial tachycardia or nodal tachycardia with an accessory path as bystander. Thus, we arrived to the diagnosis of an antidromic tachycardia with an antegrade arm constituted by an accessory pathway and the retrograde arm by the normal conduction system. The slow and decremental antegrade conduction determined the absence of ventricular preexcitation in the 12-lead EKG while in sinus rhythm. These pathways with slow and decremental conduction and with no retrograde conduction have been described in the tricuspid ring (Mahaim fibers) but very rarely in the mitral ring. ${ }^{3}$ Additionally, this patient had a slow pathway with ventricular echo that was not part of the tachycardia circuit, since it was not always induced with an AV jump, it was very easily inducible from the ventricle and because the tachycardia was not preceded by Hisian deflection, which excluded the diagnosis of nodal reentrant tachycardia (Figure 3).

Nevertheless, the case we described in this report had the particularity of being associated with left and right branch block during programmed atrial pacing with prolongation HV interval. These alterations disappeared after ablation of the accessory pathway, so they could be interpreted as a functional block resulting from the partial penetration of the impulse through the accessory pathway in the His Purkinje system. Moreover, the left bundle branch block image shows a negative QRS complex in aVL lead, which could be a fusion beat between the two wave fronts. Embryological studies in animals have shown the presence of fibers with calcium behavior in the mitral aortic continuity that could be remnants of a wider conduction system present during embryogenesis. The lack of complete regression could lead to the development of atrial tachycardias and/or reentrant antidromic tachycardias mediated with decremental behavior, as in this case. ${ }^{4}$ González R published a series of cases of atrial tachycardia in this area, with short $\mathrm{AH}$ intervals, implying a preferential input towards the $\mathrm{AV}$ node. ${ }^{5}$ The presence of bilateral functional branch block in our patient could be related to a critical penetration of 
the impulse through the accessory pathway, setting each of the branches in a relative refractory period. Proof of this is that ablation of the accessory pathway eliminated bilateral branch blockade. In conclusion, although accessory pathways with slow and decremental conduction associated with a slow pathway were described in aortic mitral continuity in unique cases, this is the first published report of a pathway with Mahaim-like behavior in aortic mitral continuity that leads to antidromic tachycardia and bilateral branch block. Identifying this mechanism is important because it can therefore prevent a possible pacemaker implant.

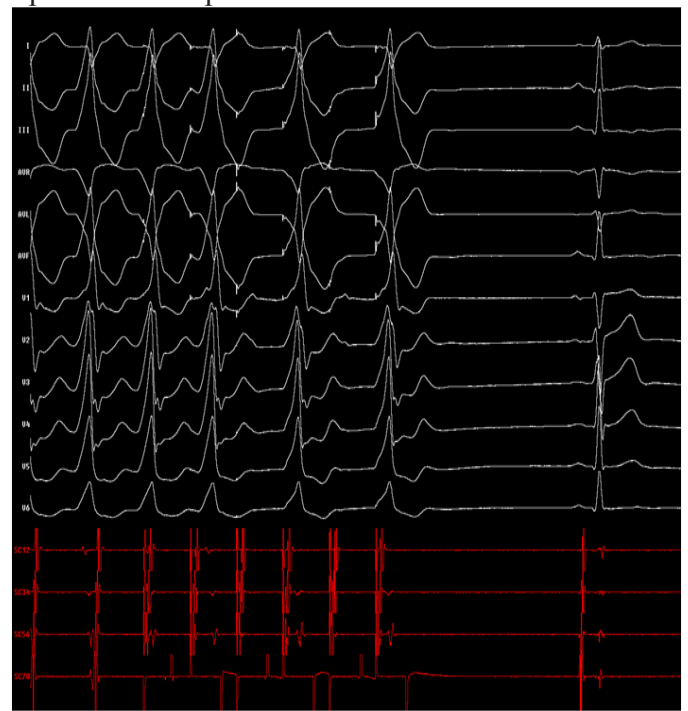

Figure 5 Atrial stimulation at high frequency terminates the tachycardia without advancing any QRS complex.

\section{References}

1. Arruda MS, McClelland JH, Wang X, et al. Development and Validation of an ECG Algorithm for Identifying Accessory Pathway Ablation Site in Wolff-Parkinson-White Syndrome. $J$ Cardiovasc Electrophysiol. 1988;9(1):2-12.

2. Wilsmore BR, Tchou PJ, Kanj M, et al. Catheter Ablation of an Unusual Decremental Accessory Pathway in the Left Coronary Cusp of the Aortic Valve Mimicking Outflow Tract Ventricular Tachycardia. Circ Arrhythm Electrophysiol. 2012;5:e104-e108

3. Sternick EB. Mahaim Fibre Tachycardia: Recognition and Management. Indian Pacing Electrophysiol J. 2003;3(2):47-59.

4. Hai JJ, Chahal AA, Friedman PA, et al. Electrophysiologic Characteristics of Ventricular Arrhythmias Arising From the Aortic Mitral Continuity Potential Role of the Conduction System. J Cardiovasc Electrophysiol. 2015;26(2):158-163.

5. Mario DG, Leonardo JC, Monique RM, et al. Left Atrial Tachycardia Originating From the Mitral Annulus-Aorta Junction. Circulation. 2004;110:3187-3192. 